\title{
REPARATIVE PROCESSES FEATURES IN TROPHIC ULCERS CAUSED BY DIABETES MELLITUS WITH THE USE OF PLATELET-RICH PLASMA
}

\author{
Igor D. Duzhiy, Andrii S. Nikolaienko, Vasyl M. Popadynets, Oleksandr V. Kravets, Igor Y. Hresko, \\ Stanislav 0. Holubnichyi, Vladyslav V. Sikora, Mykola S. Lyndin, Anatolii M. Romaniuk \\ SUMY STATE UNIVERSITY, SUMY, UKRAINE
}

\begin{abstract}
The aim: Was following: the improvement of the treatment results of the lower limbs ulcers, caused by the diabetes mellitus by using our technique of the platelet-rich plasma application; the study of the features of the morphological and immunohistochemical changes, and the effect of the growth factors of the platelet-rich plasma on the regeneration and healing of the ulcers.

Materials and methods: 38 patients with the trophic ulcers of the lower limbs, caused by diabetes mellitus were involved in the study. To assess the morphological features of the reparative processes before and after the treatment with PRP, the histological and immunohystochemical studies of the biopsy specimen of ulcers were carried out. Results: The total epithelialization of the ulcers in the patients from the main group was achieved on 46.5 day, and in the comparison group - 81.7 day. The light and optical study of the specimen of the main group, using the autologous platelet-rich plasma revealed the elimination of the destructive changes and reduction of the mixed-cellular infiltration compared to the patients from the comparison group. The immunohystochemical study revealed the increase of the growth factors receptors.

Conclusions: The usage of the autologous platelet-rich plasma in the treatment of the trophic ulcers results in the rapid regeneration by the decrease of the destructive and inflammatory changes as well as the improvement of the vascularization.
\end{abstract}

KEY WORDS: diabetes mellitus, trophic ulcer, platelet-rich plasma

\section{INTRODUCTION}

Nowadays the diabetes mellitus (DM) is one of the most important medical and social problems that concerns the most economically developed countries of the world. According to the World Health Organization, 108 million of adults worldwide suffered from DB in 1980, and in $2014-422$ million. The incidence rate has almost doubled for this period: the number of adults with diabetes has increased from $4.7 \%$ to $8.5 \%$. It can be caused by the nutritional disorders, as well as by the risk factors, linked to them, such as overweighting and obesity. Over the last decade, the incidence rate of DM has increased faster in low- and middle-income countries compared to more developed countries [1-5]. According to the International Diabetes Federation, about 592 million people will suffer from DB by 2035 [6].

Diabetic foot syndrome is one of the most serious complications of DM. Condition of microcirculatory bed is extremely important for development degenerative and necrotic changes of epithelium in DM with formation of feedback mechanism between condition of microcirculatory bed and metabolic disorders [7]. Importance of sufficient blood supplying is described in connection with endothelial function and nitric oxide synthase synthesis with different origin of necrotic changes even in immune and autoimmune process could be also explained as consequences of condition of microcirculatory bed with ulcerative process formation [8,9]. Microcirculation disorders caused by DM are characterized by significant changes in microangioarchitecture with uneven congestion, reduced specific volume of vessels, thickening of vascular walls and inflammatory changes $[7,9]$.

Development of the trophic ulcers of the lower limbs is typical manifestation of this disease, that reduces the quality of life and leads to the disability and significant financial expenses for the treatment. 16-18\% of the patients with DM suffer from the lower limbs ulceration, and $28 \%$ of cases end with the various types of amputations [2,4,10-12]. It is known that $80-90 \%$ of patients, who need a high amputation due to DM despite the long-term treatment, suffered from the chronic ulceration. Despite various treatment methods and the wide range of medications, a problem of trophic ulcers treatment still remains unsolved [4-6,13-15]. Thus, the unsatisfactory treatment of the trophic ulcers of lower limbs, as well as the significant level of disability and financial expenses for treatment prove the importance of this problem. The use of the growth factors that are contained in platelet $\alpha$-granules seems to be a promising direction in the treatment of the diabetic foot ulcers [16]. Recently, the platelet-rich plasma (PRP) enriched with the 
growth factors is offered as a substrate that stimulates the reparative processes in the tissues [17]. The concentration of the growth factors in PRP is significantly high. The degranulation of the platelet $\alpha$-granules leads to the release of the platelet-derived growth factor (PDGF), platelet-derived endothelial growth factor (PDEGF), transforming growth factor (TGF), vascular endothelial growth factor (VEGF), platelet-derived angiogenesis factor (PDAF) and epidermal growth factor (EGF). These biologically-active substances activate the proliferation of the epidermal cells and endotheliocytes, stimulate the angiogenesis, collagen synthesis and fibroblast proliferation [18-22]. Taking into account the abovementioned facts, the PRP is applied in traumatology, dentistry, surgery, and cosmetology to stimulate the tissue regeneration. However, the ways of the reparative process are insufficiently studied. The features of the ulceration, as well as its area, features of the arterial blood circulation and the presence of the related diseases have not been considered in their use [23-28].

Thus, it is obvious that the treatment of the trophic ulcers, caused by DM is an actual problem that requires the new treatment approaches for its solving.

\section{THE AIM}

Object of the study was following: the improvement of the treatment results of the lower limbs ulcers, caused by the diabetes mellitus by using our technique of the platelet-rich plasma application; the study of the features of the morphological and immunohistochemical changes, and the effect of the growth factors of the platelet-rich plasma on the regeneration and healing of the ulcers.
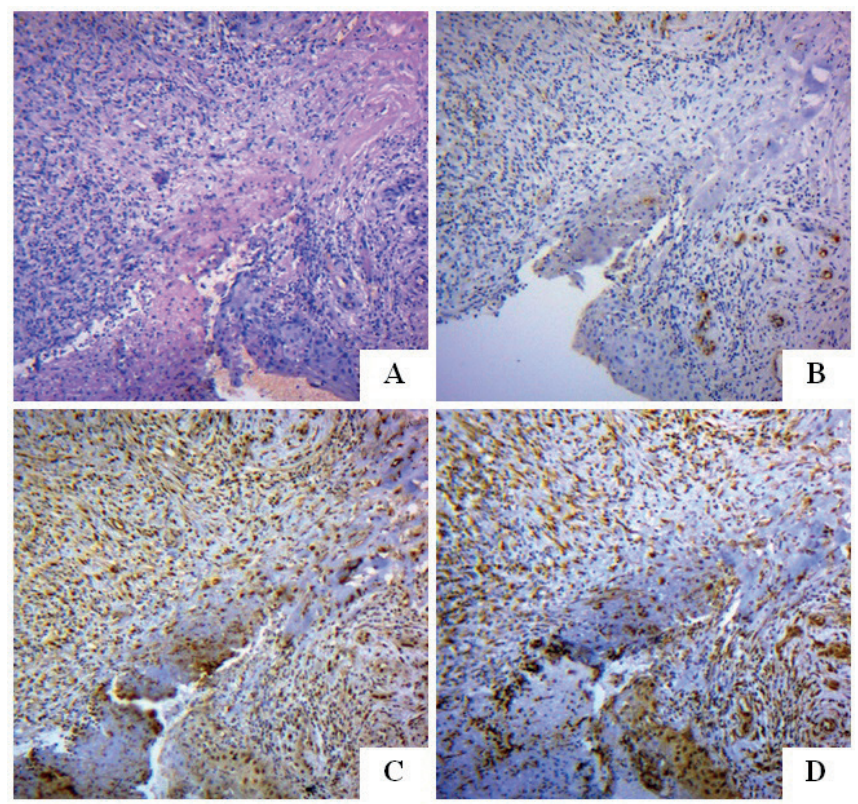

Fig 1. Fragment of the foot ulceration in the comparison group. Histological (hematoxylin and eosin staining (A)) and immunohystochemical (determination of CD31 (B), Podoplanin (C) and VEGF(D) receptors) studies of the serial tissue specimens. Magnification $\times 100$.

\section{MATERIALS AND METHODS}

38 patients with the trophic ulcers of the lower limbs, caused by DM were examined and given treatment at the vascular surgery and surgical departments of Sumy regional clinical hospital. The conservative treatment was carried out with the use of PRP. The average disease duration in the patients was more than 18 years. There were 16 males (42\%) and 22 females (58\%) patients. The patients were selected according to the following criteria: area of ulceration about $5 \mathrm{~cm}^{2}$, the duration of the ulceration more than 4 weeks and I-II stage of the trophic ulcers according to Wagner's classification (IWGDF, 2000). The patients with sepsis, acute myocardial infarction, osteoarticular apparatus affection in the area of the ulcer, critical ischemia of the lower limbs were excluded.

The patients were divided into two groups. The experimental (main) group (I) consisted of 20 patients ( 8 males and 12 females patients). After receiving the agreement their standard treatment was complemented by the usage of PRP. The comparison (II) group consisted of 18 patients ( 8 males and 10 females patients) who received the standard treatment: sanitation of the ulcers (treatment with the antiseptic, necretomy), correction of the carbohydrate exchange (antihyperglycemic drugs, insulin), antibiotics treatment (cephalosporins, fluroquinolones, metronidazoles, depending on the bacterial flora), vascular drugs (vasoprotective drugs, vasodilating agents, disaggregants), unload of the pressure from the foot with a help of orthotics.

The PRP was obtained by two-step centrifugation method. At the first step, the centrifugation lasted for 15 minutes with the rotation speed of $1000 \mathrm{rpm}$. It allowed obtain the mixture
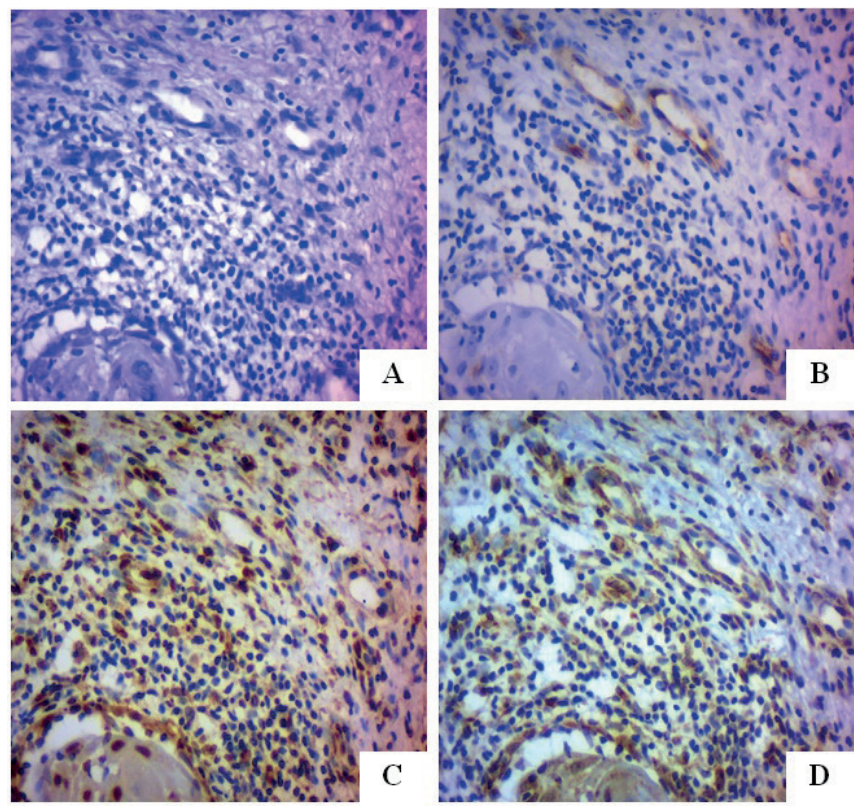

Fig 2. Fragment of the foot ulceration in the comparison group. Histological (hematoxylin and eosin staining (A)) and immunohystochemical (determination of CD31 (B), Podoplanin (C) and VEGF(D) receptors) studies of the serial tissue specimens. Magnification $\times 400$. 

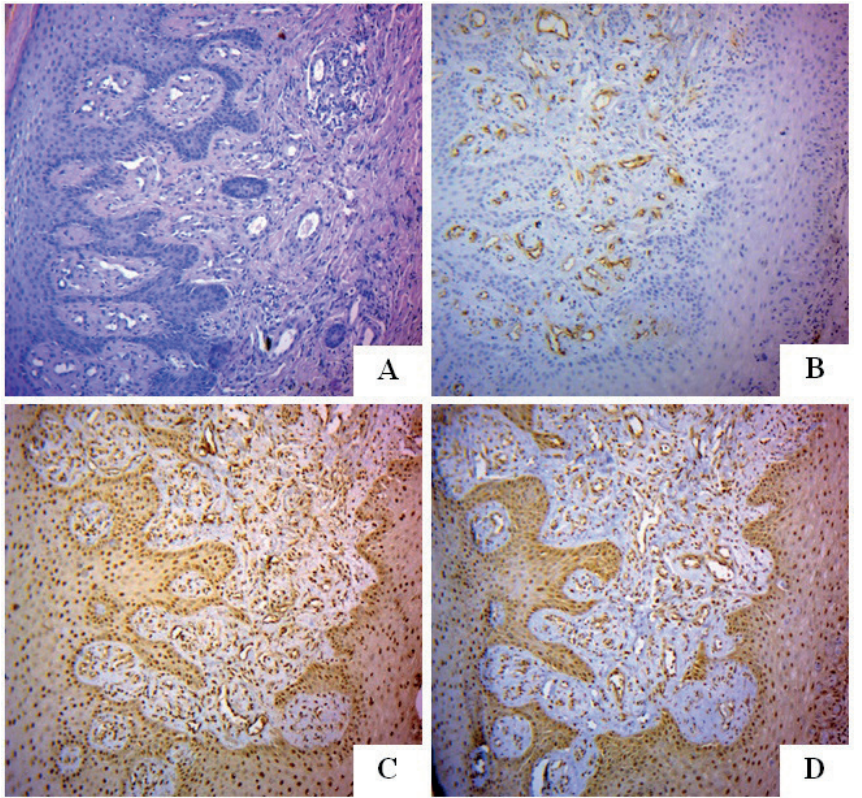

Fig 3. Fragment of the foot ulceration in the main group. Histological (hematoxylin and eosin staining (A)) and immunohystochemical (determination of CD31 (B), Podoplanin (C) and VEGF(D) receptors) studies of the serial tissue specimens. Magnification $\times 100$.
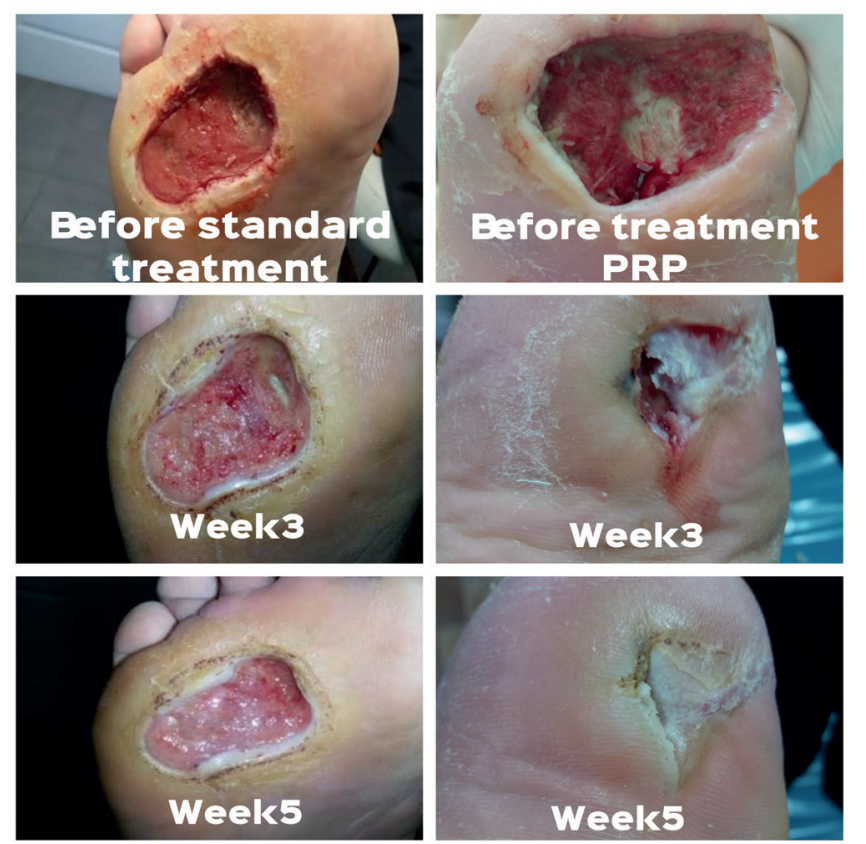

Fig 5. Dynamics of trophic ulcer healing under standard treatment and using PRP.

of plasma with the low concentration of the platelet. At the second step, the tubes with the mixture of the top and middle layers of the obtained concentrate have been centrifuged at $1700 \mathrm{rpm}$ for 10 minutes. The bottom layer of the obtained mixture was collected in a separate sterile syringe. The fraction that contained the largest number of platelets was used for the treatment. A series of 5-10 injections of $0.2 \mathrm{ml}$ of PRP, activated with the $10 \%$ solution of $\mathrm{CaCl}$ were given around the trophic ulcer at $10 \mathrm{~mm}$ from its borders. The injections
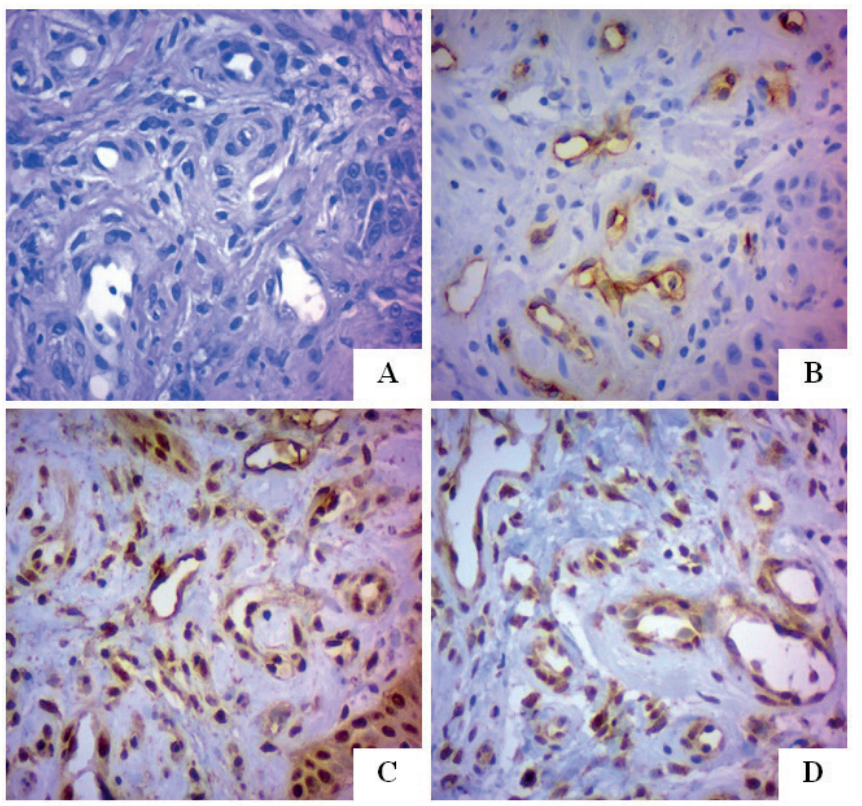

Fig 4. Fragment of the foot ulceration in the main group. Histological (hematoxylin and eosin staining $(A)$ ) and immunohystochemical (determination of CD31 (B), Podoplanin (C) and VEGF(D) receptors) studies of the serial tissue specimens. Magnification $\times 400$.

were made on $5^{\text {th }}, 10^{\text {th }}$ and $20^{\text {th }}$ day of the treatment. This technique was supplemented by the local PRP applications that were carried out during 15 days at the intervals of 2 days.

To assess the morphological features of the reparative processes in the soft tissues in the area of the ulcers before and after PRP treatment, the histological study of the biopsy samples, collected on the edge of the healthy tissue - the ulcers, by using hematoxylin and eosin staining method was carried out. The epidermization, vascularization and inflammatory infiltration of the ulcerative defect were assessed during the study.

Taking into account the blood supply peculiarities in the regenerating tissue and release of the VEGF that influence the healing speed, the immunohystochemical study of the following receptors was carried out: CD31 (clone 1A10, dilution 1 : 50) that is the marker of the existing and newly formed vessels, Podoplanin (clone 4D5aE5E6, dilution $1: 200$ ) the marker of the lymphatic vessels and VEGF (polyclonal, dilution $1: 200$ ) the marker of vessel endothelial growth.

The ulceration area was measured by using the Kundin method. Thus, the length and the width of the ulcers were assessed in such way that the biggest obtained measures were located on the perpendicular axis. The area was calculated by the following formula: $\mathrm{S}=\mathrm{L} \times \mathrm{W} \times 0.785$, where $\mathrm{L}$ is the length of the ulcer and $\mathrm{W}$ - the ulcer's width.

Statistical processing of the results was carried out by using the Student's t-test. The difference between the mean values at $\mathrm{p}<0.05$ was taken as a reliable value.

\section{RESULTS}

The morphological studies of the samples, stained with hematoxylin and eosin revealed the different regeneration of the trophic ulcers at different periods. In the main group, 


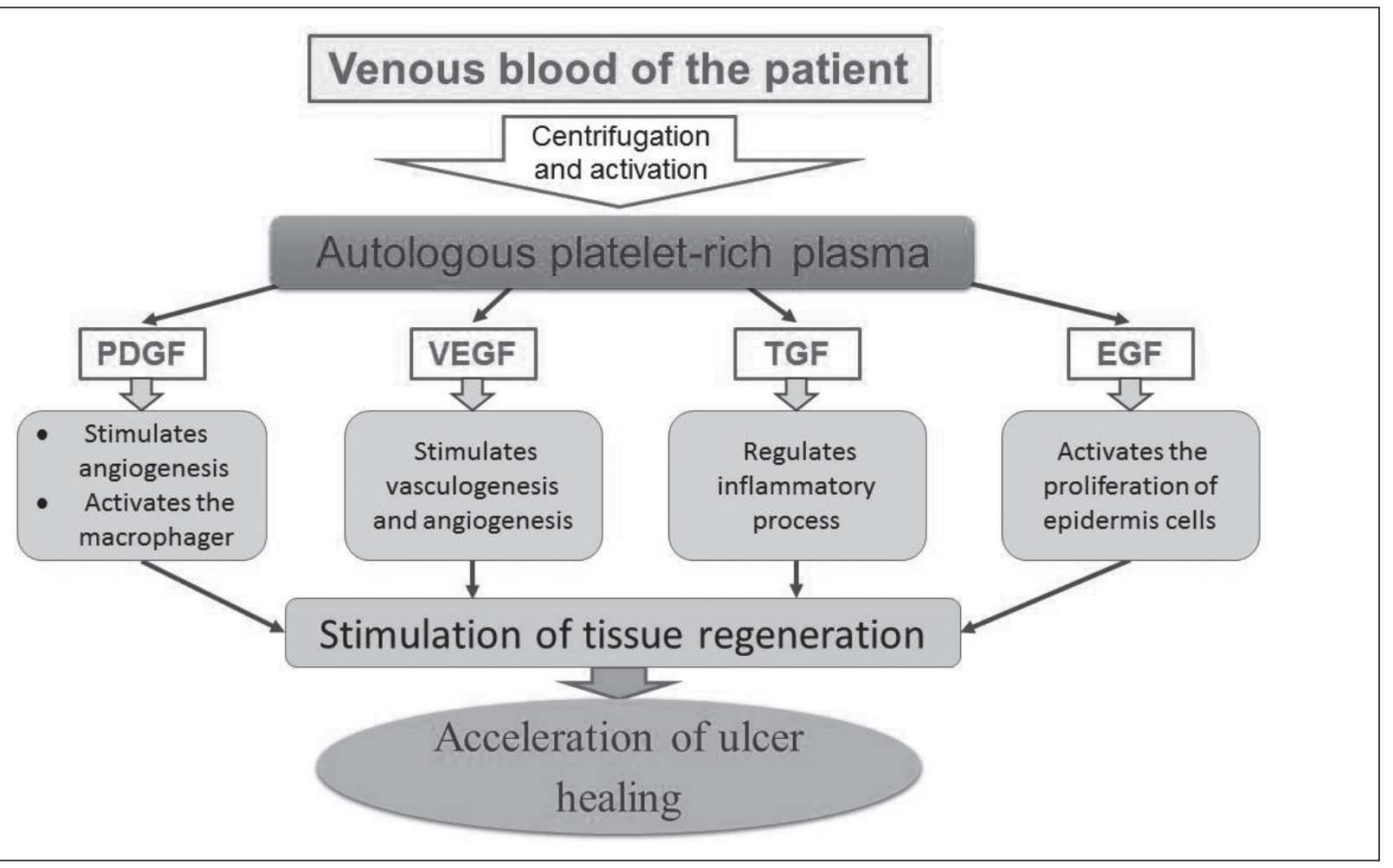

Fig 6. The scheme the effectiveness of the trophic ulcers treatment with the use of platelet-rich plasma.

the rapid epithelialization of the defect, "maturation" of the granulation tissue, elimination of the destructive changes as well as the reduction of the mixed-cellular inflammatory infiltration was observed due to the use of PRP, compared to the patients from the comparison group. (Fig. 1-4- A).

During the immunohistochemical study, the denser vascularization both in the regenerating tissue and surrounding areas was revealed in the main group (CD 31-positive cells). The vascular component was presented by the capillaries, venules and arterioles, located in the granulation tissue and surrounding areas (Fig. 1-4- B).

The study of Podoplanin receptors allowed visualize the lymphatic vessels. Their number was significantly higher in the samples, obtained from the patients which used PRP (Fig. 1-4- C).

The immunohistochemical study of VEGF receptors revealed endothelial expression in the regenerating tissue in the patients from the main group. Besides, some parts of cells of the stromal component of the regenerator were VEGF- positive. The number of these receptors was significantly lower in the comparison group that indicates the prevalent angiogenic potential of PRP (Fig. 1-4- D).

We considered the following clinical indicators of the ulcer healing: the intensity of the inflammation, formation of the granulation tissue and the epithelialization. In first 2 weeks of the treatment, the inflammation was less intensive and the growth of the granulation tissue was activated that led to the healing of the ulcers. In the main group, the trophic ulcers decreased by $60.5 \%$ on the $30^{\text {th }}$ day of the treatment. The damage area in the comparison group was reduced by $39.6 \%$ for this period, that is 1.5 times less than in the main group $(p<0.05)$. The total epithelialization of the ulcers in the patients from the main group was achieved on 46.5 day and in the comparison group - on 81.7 day ( $p$ $<0.05$ ). Based on the obtained results the healing of the ulcers of the patients from the main group is faster in 1.7 times $(\mathrm{p}<0.05)$. The average period of the hospitalization of the patients from the main group was 14.6 days and for the comparison group -23.6 days $(\mathrm{p}<0.05)$. The criteria for the discharge of the patients were the following: cleaning of the trophic ulcer, reduced inflammation, granulation tissue growth and beginning of the epithelialization.

\section{DISCUSSION}

The main principles of the treatment of the trophic ulcer, caused by the DM were provided in "International agreement on diabetic foot". It is a multivariate approach that includes the following treatment: normalization of carbohydrate metabolism, unload of the foot with orthopedic insoles, prevention and control of infection, ulcer treatment (vacuum therapy), improvement of blood flow etc., aimed at acceleration of the ulcer healing $[13,15]$.

Standard treatment guidelines are increasingly being supplemented with new immunologically safe therapeutic technologies. Among them, the use of autologous blood components is a modern promising area of medicine [29]. In our study we applied the combined standard treatment of the trophic ulcers, caused by the DM, with the use of PRP. Positive effect of treatment was achieved: reduced inflammation, acceleration of the ulcers epithelization. Thus, in patients who were treated with this technique, the healing of the ulcers 
was much faster in comparison with the patients treated under the standard scheme (Figure 5). The assessment of the morphological changes revealed the accelerated regeneration, maturation of granulation tissue and reduced mixed-cell inflammatory infiltration due to the use of PRP. Immunohistochemical research has shown that the additional use of PRP increases the number of cells that express the VEGF receptors in regenerating tissues. The angiogenic properties of this protein promote the increased vascular growth, as well as the increase of their density (CD31-posivite structures). Among them, significant parts fall to the lymphatic vessels (Podoplanin-positive structures). Endothelial proliferation, increased angiogenesis and lymphatic vessels growth lead to the improvement of regeneration of the tissues, both due to the restoration of blood flow in hypoxia-affected areas and normalization of the lymphatic drainage system (Figure 6).

Based on the study results, the use of PRP is a simple, effective and rather safe method to improve the healing of the diabetic ulcers. A significant number of the growth factors (vascular, thrombocytic, epidermal) and immunoglobulins not only increases the epithelization rate of the trophic ulcers but also stimulate the local, anti-inflammatory and immune response of the body.

\section{CONCLUSIONS}

Platelet-rich plasma, used in complex treatment of the diabetic trophic ulcers, accelerates the regeneration of the skin defect by the reduction of the destructive and inflammatory changes and improvement of the vascularization. This reduces the period of the trophic ulcers epithelialization in 1.7 times and period of hospitalization of the patients up to 14.6 days compared to the comparison group $(\mathrm{p}<0.05)$. The immunohistochemical study revealed that platelet-rich plasma as a source of VEGF, stimulates the vascularization dense of the regenerated tissues through the appearance of the capillaries, venules, arterioles and lymphatic vessels. This intensifies the positive effect of ulcers healing.

\section{REFERENCES}

1. World Health Organization Global report on diabetes. 2016. http:// www.who.int/diabetes/global-report/en/

2. Chentli F., Azzoug S., Mahgoun S. Diabetes mellitus in elderly. Indian J Endocrinol Metab. 2015; 19(6): 744-752. doi: 10.4103/22308210.167553

3. Unnikrishnan R., Pradeepa R., Joshi S.R., Mohan V. Type 2 Diabetes: Demystifying the Global Epidemic. Diabetes 2017; 66(6): 1432-1442. doi: $10.2337 / \mathrm{db} 16-0766$

4. Kakkar R. Rising burden of Diabetes-Public Health Challenges and way out. Nepal J Epidemiol. 2016; 6(2): 557-559. doi: 10.3126/nje. v6i2.15160

5. Wang M., Hu R.Y., Wang H., Gong W.W., et al. Age at natural menopause and risk of diabetes in adult women: Findings from the China Kadoorie Biobank study in the Zhejiang area. J Diabetes Investig. 2018; 9(4): 762-768. doi: 10.1111/jdi.12775

6. Cho N.H., Shaw J.E., Karuranga S., Huang Y. et al. IDF Diabetes Atlas: Global estimates of diabetes prevalence for 2017 and projections for 2045. Diabetes Res Clin Pract. 2018;138:271-281. doi: 10.1016/j. diabres.2018.02.023.
7. Denga 0., Pyndus T., Gargin V., Schneider S. Influence of metabolic syndrome on condition of microcirculatory bed of oral cavity. Georgian Med News. 2017; 273:99-104.

8. Nazaryan R.S., Kryvenko L.S., Gargin V.V. The role of nitric oxide synthase in the modulation of the immune response in atopic disease. The New Armenian Medical Journal. 2017;11(2):52-57.

9. Kovach I., Kravchenko L., Khotimska Y., Nazaryan R. et al. Influence of ozone therapy on oral tissue in modeling of chronic recurrent aphthous stomatitis. Georgian Med News. 2017;(264):115-119.

10. Bakker K., Apelqvist J., Lipsky B.A., Van Netten J.J. International Working Group on the Diabetic Foot. The 2015 IWGDF guidance documents on prevention and management of foot problems in diabetes: development of an evidence-based global consensus. Diabetes Metab Res Rev. 2016;32(1):2-6. doi: 10.1002/dmrr.2694.

11. Hwang D.J., Lee K.M., Park M.S., Choi S.H., et al. Association between diabetic foot ulcer and diabetic retinopathy. PLoS One. 2017;12(4):0175270. doi: 10.1371/journal.pone.0175270

12. Oksuz E., Malhan S., Sonmez B., Tekin R.N. Cost of illness among patients with diabetic foot ulcer in Turkey. World J Diabetes. 2016; 7(18): 462-469. doi: 10.4239/wjd.v7.i18.462

13. Boulton A.J.M. The diabetic foot. Medicine. 2015;43(1):33-37. doi: 10.1016/j.mpmed.2014.10.006

14. Alexiadou K., Doupis J. Management of Diabetic Foot Ulcers. Diabetes Ther. 2012; 3(1): 4. doi: 10.1007/s13300-012-0004-9

15. Armstrong D.G.1., Lavery L.A., Wu S., Boulton A.J. Evaluation of removable and irremovable cast walkers in the healing of diabetic foot wounds: a randomized controlled trial. Diabetes Care. 2005;28(3):551-4.

16. Marx R.E. Platelet-rich plasma (PRP): what is PRP and what is not PRP? Implant Dent. 2001;10(4):225-8.

17. El-Sayed A. Abd El-Mabood, Hazem E. Ali. Platelet-rich plasma versus conventional dressing: does this really affect diabetic foot woundhealing outcomes? The Egyptian Journal of Surgery 2018;37(1):16-26. doi: 10.4103/ejs.ejs_83_17

18. Foster T.E., Puskas B.L., Mandelbaum B.R., Gerhardt M.B. et al. Plateletrich plasma: from basic science to clinical applications. Am J Sports Med. 2009;37(11):2259-72. doi: 10.1177/0363546509349921.

19. Wen Y.H., Lin W.Y., Lin C.J., Sun Y.C. et al Sustained or higher levels of growth factors in platelet-rich plasma during 7-day storage. Clin Chim Acta. 2018;483:89-93. doi: 10.1016/j.cca.2018.04.027.

20. Etulain J., Mena H.A., Meiss R.P., Frechtel G. et al. An optimised protocol for platelet-rich plasma preparation to improve its angiogenic and regenerative properties. Scientific Reports. 2016;8:1513. 21.

21. Chicharro-Alcántara D.,Rubio-ZaragozaM.,Damiá-GiménezE.,Carrillo-Poveda J.M. et al. Platelet Rich Plasma: New Insights for Cutaneous Wound Healing Management. J Funct Biomater. 2018;9(1):10. doi: 10.3390/ffb9010010.

22. Romaniuk A., Lyndin M., Moskalenko R., Kuzenko Y. et al Pathogenetic mechanisms of heavy metals effect on proapoptotic and proliferative potential of breast cancer. Interv Med Appl Sci. 2015;7(2):63-8. doi: 10.1556/1646.7.2015.2.4

23. Alavi S., Eshghi F.P., Arzhangian H. Autologous Platelet-Rich Plasma for Healing of an Oncologic Dehisced Wound. APSP J Case Rep. 2013;4(3):44.

24. Reddy S.H., Reddy R., Babu N.C., Ashok G.N. Stem-cell therapy and platelet-rich plasma in regenerative medicines: A review on pros and cons of the technologies.J Oral Maxillofac Pathol. 2018;22(3):367-374. doi: 10.4103/jomfp.JOMFP_93_18

25. Garg S., Manchanda S. Platelet-rich plasma—an'Elixir' for treatment of alopecia: personal experience on 117 patients with review of literature. Stem Cell Investig. 2017; 4: 64. doi: 10.21037/sci.2017.06.07 
26. Duzhiy I., Popadynets V., Nikolaienko A., Lyndin M. et al. Hypertensive ulcer of lower extremity (Martorell's syndrome): clinical case with the treatment improvement. Bangladesh Journal of Medical Science. 2017;16(2):325-328. doi: 10.3329/bjms.v16i2.31212

27. Jalaluddin M., Singh D.K., Jayanti I., Kulkarni P. et al. Use of Platelet Rich Plasma in the Management of Periodontal Intra-Osseous Defects: A Clinical Study. J Int Soc Prev Community Dent. 2017; 7(2): 105-115. doi: 10.4103/jispcd.JISPCD_28_17

28. Bus S.A., Armstrong D.G., van Deursen R.W., Lewis J.E. et al. International Working Group on the Diabetic Foot. IWGDF guidance on footwear and offloading interventions to prevent and heal foot ulcers in patients with diabetes. Diabetes Metab Res Rev. 2016;32(1):25-36. doi: 10.1002/ dmrr.2697.

29. Hirase T., RuffE., Surani S., Ratnani I. Topical application of platelet-rich plasma for diabetic foot ulcers: A systematic review. World J Diabetes. 2018;9(10):172-179.

\section{ORCID and contributionship:}

Igor D. Duzhiy: 0000-0002-4995-0096 E, F

Andrii S. Nikolaienko: 0000-0002-6766-144X A, B, D, E

Vasyl M. Popadynets: 0000-0002-9727-0909 A, B, D, E

Oleksandr V. Kravets: 0000-0003-3394-6691 B, C

Igor Y. Hresko: 0000-0002-6092-135X B, C
Stanislav O. Holubnichyi: 0000-0002-4965-0114 ${ }^{B, C}$

Vladyslav V. Sikora: 0000-0002-4147-6879 A, B, D

Mykola S. Lyndin: 0000-0003-4385-3903 A, B, D, E

Anatolii M. Romaniuk: 0000-0003-2560-1382 E, F

\section{Conflict of interest:}

The Authors declare no conflict of interest.

\section{CORRESPONDING AUTHOR Vladyslav V. Sikora \\ Sumy State University \\ 31 Privokzalnaya st., 40022 Sumy, Ukraine \\ tel: +380507276193 \\ e-mail:v.sikora@med.sumdu.edu.ua}

Received: 24.10.2019

Accepted: 27.08 .2020

A - Work concept and design, B - Data collection and analysis, C - Responsibility for statistical analysis,

D-Writing the article, $\mathbf{E}$-Critical review, $\mathbf{F}$ - Final approval of the article 